\title{
Relationship between paediatric CT scans and subsequent risk of leukaemia and brain tumours: assessment of the impact of underlying conditions
}

\author{
Amy Berrington de Gonzalez ${ }^{*},{ }^{\text {, Jane A Salotti }}{ }^{2}$, Kieran McHugh ${ }^{3}$, Mark P Little ${ }^{1}$, Richard W Harbron ${ }^{2}$, \\ Choonsik Lee ${ }^{1}$, Estelle Ntowe ${ }^{1}$, Melissa Z Braganza ${ }^{1}$, Louise Parker ${ }^{4}$, Preetha Rajaraman ${ }^{1}$, Charles Stiller $^{5}$, \\ Douglas R Stewart ${ }^{1}$, Alan W Craft ${ }^{2}$ and Mark S Pearce ${ }^{2}$ \\ ${ }^{1}$ Radiation Epidemiology Unit, Division of Cancer Epidemiology and Genetics, NCI, Bethesda, MD, USA; ${ }^{2}$ Institute of Health and \\ Society, Newcastle University, Sir James Spence Institute, Royal Victoria Infirmary, Newcastle upon Tyne NE1 4LP, UK; ${ }^{3}$ Great \\ Ormond Street Hospital for Children NHS Trust, London WC1N 3JH, UK; ${ }^{4}$ Departments of Medicine and Paediatrics, Population \\ Cancer Research Program, Dalhousie University, Halifax, Nova Scotia, Canada and ${ }^{5}$ New College, University of Oxford, Oxford, UK
}

Background: We previously reported evidence of a dose-response relationship between ionising-radiation exposure from paediatric computed tomography (CT) scans and the risk of leukaemia and brain tumours in a large UK cohort. Underlying unreported conditions could have introduced bias into these findings.

Methods: We collected and reviewed additional clinical information from radiology information systems (RIS) databases, underlying cause of death and pathology reports. We conducted sensitivity analyses excluding participants with cancer-predisposing conditions or previous unreported cancers and compared the dose-response analyses with our original results.

Results: We obtained information from the RIS and death certificates for about 40\% of the cohort ( $n \sim 180000)$ and found cancerpredisposing conditions in 4 out of 74 leukaemia/myelodysplastic syndrome (MDS) cases and 13 out of 135 brain tumour cases. As these conditions were unrelated to CT exposure, exclusion of these participants did not alter the dose-response relationships. We found evidence of previous unreported cancers in 2 leukaemia/MDS cases, 7 brain tumour cases and 232 in non-cases. These previous cancers were related to increased number of CTs. Exclusion of these cancers reduced the excess relative risk per mGy by $15 \%$ from 0.036 to 0.033 for leukaemia/MDS $(P$-trend $=0.02)$ and by $30 \%$ from 0.023 to $0.016(P$-trend $<0.0001)$ for brain tumours. When we included pathology reports we had additional clinical information for $90 \%$ of the cases. Additional exclusions from these reports further reduced the risk estimates, but this sensitivity analysis may have underestimated risks as reports were only available for cases.

Conclusions: Although there was evidence of some bias in our original risk estimates, re-analysis of the cohort with additional clinical data still showed an increased cancer risk after low-dose radiation exposure from CT scans in young patients.

Computed tomography (CT) scan use has increased substantially around the world since its widespread introduction in the $1980 \mathrm{~s}$ (Mettler et al, 2009). Indisputably, CT scans have the ability to save lives in injury management, by detecting disease early and avoiding unnecessary operations. These benefits need to be balanced against a potential increase in the risk of radiation-related cancer, particularly in children who are more radiosensitive and who, especially in the past, received higher radiation doses

*Correspondence: Dr A Berrington de Gonzalez; E-mail: Berringtona@mail.nih.gov

Received 30 April 2015; revised 10 September 2015; accepted 1 November 2015

(c) 2016 Cancer Research UK. All rights reserved 0007-0920/16 
(Frush and Goske, 2015). It is difficult to assess cancer risks from low-dose radiation directly because of the need for large populations, detailed exposure information and long-term followup to study this question effectively. In a study of UK paediatric CT scan use, we reported evidence of a dose-response relationship between the risk of leukaemia and brain tumours and organspecific ionising radiation exposure (Pearce et al, 2012a). The dose-response relationship was broadly compatible with that observed in the Japanese atomic bomb survivors. In our previous study, we examined the possibility that our results were influenced by reverse causation, that is the CT scan was taken because of preexisting disease and therefore not a cause, by extending the exclusion period between CT scan and cancer diagnosis from 5 to 10 years, but this did not decrease our dose-response estimate. We also considered the possibility that the children may have underlying conditions that predispose to cancer, but as these conditions are very rare and not clearly related to frequency of CT scanning, we thought this was unlikely to have biased our findings (Pearce et al, 2012b).

Our retrospective cohort study was based on record linkage between radiology databases from hospitals and cancer registration data and includes approximately 180000 children and young adults. To directly investigate concerns about the impact of underlying conditions (Pearce et al, 2012b), we collected and reviewed additional clinical information to assess whether the children had cancer-predisposing conditions or whether the CT scan may have been performed because of a preexisting or unreported cancer. As no single electronic database exists in the United Kingdom with all of the relevant clinical information, we collected and reviewed information from the available radiologists' contemporaneous comments from the hospital databases, underlying cause of death and pathology reports. We then conducted sensitivity analyses where we excluded these patients with cancerpredisposing conditions or preexisting cancers and compared the dose-response analyses with our original results.

\section{MATERIALS AND METHODS}

We previously published a detailed description of the methods we used to construct our retrospective cohort of approximately 180000 children who had had CT scans (Pearce et al, 2012a). Briefly, it is a record linkage study of CT scan exposure information from hospital radiology departments in 81 National Health Service (NHS) regional services in Great Britain linked to outcome information from the National Health Services Central Register (NHSCR). From each of the radiology departments, we requested information on all CT scans performed before 2003 on children and young adults (aged $<22$ years). The details included date scanned, body region scanned, name, sex and date of birth. The NHSCR used the names, dates of birth, sex and location information to link to information on cancer registrations, deaths and emigrations.

Evidence of cancer-predisposing conditions. We used three additional sources of information to assess whether the patients (cases and non-cases) had underlying conditions that are related to, or possibly related to, an increased risk of leukaemia or brain tumours: (1) text comments written by radiologists in the radiology information systems (RIS) databases that were available from about one-third of the hospitals in our study that typically described the result of the exam and noted underlying conditions, (2) underlying cause of death for those who had died, and (3) pathology reports from the appropriate Cancer Registry or hospital of diagnosis, for each of the cases of leukaemia/MDS or brain tumours. These sources of information were reviewed by clinical experts (AC, CS, KM, DS), and conditions were coded as either being related to the cancer of interest, possibly related or unrelated.

The leukaemia-predisposing conditions were (Supplementary Table A): Down syndrome (Seewald et al, 2012), Noonan syndrome (Lindor et al, 2008), Li-Fraumeni syndrome (Lindor et al, 2008), Wiskott-Aldrich syndrome (Bosticardo et al, 2009), Langerhans cell histiocytosis (Egeler et al, 1993), Kostmann granulocytopenia (Ward and Dale, 2009), neurofibromatosis type 1 (Lindor et al, 2008), ataxia telangiectasia (Lindor et al, 2008), Shwachman-Diamond syndrome (Burroughs et al, 2009), Bloom syndrome (Lindor et al, 2008), myelodysplasia monosomy 7 (Liew and Owen, 2011), Fanconi anaemia (Lindor et al, 2008) and solid organ and bone marrow (hematopoietic stem cell) transplants (Metayer et al, 2003; Engels et al, 2011) In addition, some conditions were classified as possibly related to leukaemia, including immunodeficiency syndromes (Shiels et al, 2011) and Klinefelter syndrome (Machatschek et al, 2004) The brain tumourrelated conditions were: Li-Fraumeni syndrome (Lindor et al, 2008), tuberous sclerosis (Lindor et al, 2008), neurofibromatosis type 1 and type 2 (Lindor et al, 2008), nevoid basal cell syndrome (Lindor et al, 2008), Von Hippel Lindau (Lindor et al, 2008) and Turcot syndrome (Lindor et al, 2008).

Evidence of previous cancer or possible previous cancer. Patients were ineligible for the original analysis if they had a cancer registration before the date of the first CT scan. This exclusion was performed to minimise confounding owing to previous cancer. For example, a previous cancer treated with chemotherapy or radiotherapy would be associated with an increased leukaemia risk (Churpek and Larson, 2013) and possibly more CT scans. The UK cancer registry data is reported to be at least 90\% complete (Dickinson et al, 2001, Hawkins and Swerdlow, 1992). During our review of the additional data sources described above, we did find some reports of previous cancers that had not been captured by the NHSCR. We therefore also performed a systematic review of all available additional clinical data for previous cancers. A radiologist (KM) also reviewed the radiologist's comments to determine whether there was a possible undiagnosed cancer or whether the CT could have been performed owing to cancer-related symptoms. These were categorised as 'possible previous cancers'.

Statistical analysis. Details of the original statistical analysis are provided in our previous publication (Pearce et al, 2012a). In brief, we analysed the relationship between estimated organ dose and cancer risk using Poisson regression. All tests are two sided, using the likelihood ratio test, and confidence intervals are derived using the profile likelihood except when this did not converge when the Wald-based confidence limit was used instead. We used a dose lagging period of 2 years for leukaemia and 5 years for brain tumours. To reduce the likelihood of reverse causation, we started follow-up 2 years for leukaemia, and 5 years for brain tumours, after the date of first CT scan.

To assess the possible impact of cancer-predisposing conditions, we re-analysed the dose-response relationship excluding patients (cases and non-cases) with the conditions listed above. We had to exclude rather than censor patients who were found to have a previous (unreported) cancer, because we did not have the date of diagnosis for these prior cancers and could not always confirm whether the cancer was diagnosed before or after the CT scans. Our primary set of exclusions was based on information from the radiologists' comments and death certificates, because these were equally available for cases and non-cases. We also conducted sensitivity analyses where we only used radiologists' comments to define exclusions and assessed the impact of excluding additional cases who had underlying conditions or previous cancers that were ascertained from the pathology reports. We compared the results 
from these sensitivity analyses with those from our original analysis (Pearce et al, 2012a).

To assess reverse causation, we excluded individuals for whom there was evidence that the cancer was possibly or likely evident before the diagnosis date based on the review of the radiologists' comments, that is, 'possible previous cancers'.

\section{RESULTS}

Radiologists' comments were available for about $40 \%$ of the patients (Table 1). There was no relationship between age at CT scan or year of scan or type of hospital (specialist/general) and availability of these comments. The key determinant was the hospital as some had routinely used this field on the database and others had not. When including death certificate information and pathology reports additional clinical information was available for $86 \%$ of the leukemia/MDS cases and $87 \%$ of the brain tumour cases.

From the RIS and death certificates, we found four out of 74 leukaemia/MDS cases had underlying conditions that are known to predispose to leukaemia and an additional three patients with conditions were identified from the pathology reports (Table 2). We found four cases who had had a cancer before the reported diagnosis of leukaemia, including two identified only from the pathology reports. Three of these were previous lymphomas or leukaemias, suggesting that the reported leukaemia was a relapse rather than the first diagnosis.

Our review of the non-cases $(n=178528)$ revealed that 104 of the patients with available clinical information had a leukaemiapredisposing condition (Table 2). In addition, 236 had cancers that had not been reported to the NHSCR.

The mean estimated red bone marrow (RBM) dose from the CT scans in the cases with leukaemia-predisposing conditions was similar to those without (Table 2). Exclusion of the patients with leukaemia-predisposing conditions, therefore, did not materially alter the dose-response relationship (Table 3 and Figure 1B). When exclusions were based on the comparable data for cases and non-case (RIS and death certificates), the excess relative risk (ERR) per mGy changed from 0.036 to 0.037 (95\% CI: $0.005-0.126$ ), $P$-trend $=0.01$.

The mean RBM dose in the leukaemia/MDS cases with a previous unreported cancer was considerably higher than those without (Table 2). Exclusions based on comparable data (RIS and death certificates) reduced the estimated ERR per mGy from 0.036 to 0.033 (0.004 to 0.114 ), $P$-trend $=0.02$ (Table 3 and Figure $1 C$ ). Additional exclusion of the patients with conditions that were possibly related to leukaemia or possible previous cancers had minimal additional impact on the risk coefficients. Results of analyses where exclusions were based only on the RIS data were very similar. When we also excluded previous cancers that were identified on the pathology reports for the leukaemia cases, the ERR per mGy was further reduced to 0.020 (95\% CI: -0.011 to $0.086), P$-trend $=0.13$. However, a RBM dose of $30+\mathrm{mGy}$ compared with $<5 \mathrm{mGy}$ was still associated with a significantly increased risk of leukaemia/MDS ( $\mathrm{RR}=2.63,95 \% \mathrm{CI}$ : 1.09 to 6.24 ).

The review of the radiologists' comments for the 29 leukaemia/ MDS cases with this information did not reveal any evidence that the CT scans were performed because of symptoms related to leukaemia. The indication for the CTs $(2+$ years before diagnosis $)$ included suspected hydrocephalus, headaches, meningitis, suspected congenital cerebral anomalies, congenital heart disease, limb trauma, juvenile arthritis, respiratory disorders, lymphoedema and amenorrhoea.

We found 13 of the 135 brain tumour cases had underlying conditions that are known to be related to brain tumour risk from the RIS data and death certificates, and an additional 16 were identified only from the pathology reports. There were also 10 cases who had had a cancer before the reported diagnosis of the brain tumour (three identified only from the pathology reports) and most of these were previous brain tumours (Table 4). In addition, there was evidence of a possible preexisting brain tumour $>5$ years before the reported brain tumour in 16 of the brain tumour cases based on the review of the radiologists' comments.

Our review of the non-cases $(n=176447)$ revealed that 100 patients with available clinical information had conditions that predispose to brain tumours (Table 4). In addition, 257 had a previous cancer or possible previous cancer.

The mean estimated brain dose from the CT scans in the cases with brain tumour-predisposing conditions was slightly higher in cases with, than in those without, these predisposing conditions (Table 4). Exclusion of the patients with those conditions reduced the ERR per mGy slightly from 0.023 to 0.019 (95\% CI: $0.008-$ 0.043 ), $P$-trend $<0.0001$ (Table 5 and Figure $2 \mathrm{~B}$ ).

As with the leukaemia cases, the mean brain dose in the brain tumour cases with a previous unreported cancer was higher than in those without (Table 5). After exclusion of previous cancers using data from comparable sources for cases and non-cases (RIS and death certificate), the ERR per $\mathrm{mGy}$ was reduced from 0.023 to 0.016 (95\% CI: $0.006-0.037$, $P$-trend $<0.0001$; Table 3 and Figure $2 \mathrm{C}$ ). It was further reduced by the exclusion of cases for whom there was earlier suspicion of the brain tumour in the radiologists' comments, to 0.012 (95\% CI: $0.004-0.031$ ), but the trend remained highly statistically significant $(P<0.0001)$. Results were very similar when we only excluded patients using the RIS data. However, when previous cancers or possible cancers identified on pathology reports were also excluded the ERR per $\mathrm{mGy}$ decreased further to 0.010 (95\% CI: 0.002-0.026), $P$-trend $=0.002$.

These exclusions did not alter the patterns with age at exposure or time since exposure (Supplementary Tables B and C).

\section{DISCUSSION}

After detailed review of the available clinical information, we found no evidence that patients with cancer-predisposing conditions

Table 1. Additional sources of clinical information for leukaemia/MDS cases, brain tumours and non-cases

\begin{tabular}{|c|c|c|c|c|c|c|c|c|}
\hline & \multicolumn{4}{|c|}{ Leukaemia/MDS } & \multicolumn{4}{|c|}{ Brain tumours } \\
\hline Pathology reports & 46 & $62.16 \%$ & NA & - & 89 & $65.93 \%$ & NA & - \\
\hline RIS & 29 & $39.19 \%$ & 67100 & $37.59 \%$ & 56 & $41.48 \%$ & 66162 & $37.50 \%$ \\
\hline $\begin{array}{l}\text { No additional } \\
\text { information }\end{array}$ & 10 & $13.51 \%$ & 110081 & $61.66 \%$ & 18 & $13.33 \%$ & 109331 & $61.96 \%$ \\
\hline
\end{tabular}


Table 2. Description of the leukaemia-related conditions, previous cancers and other conditions in the leukaemia/MDS cases

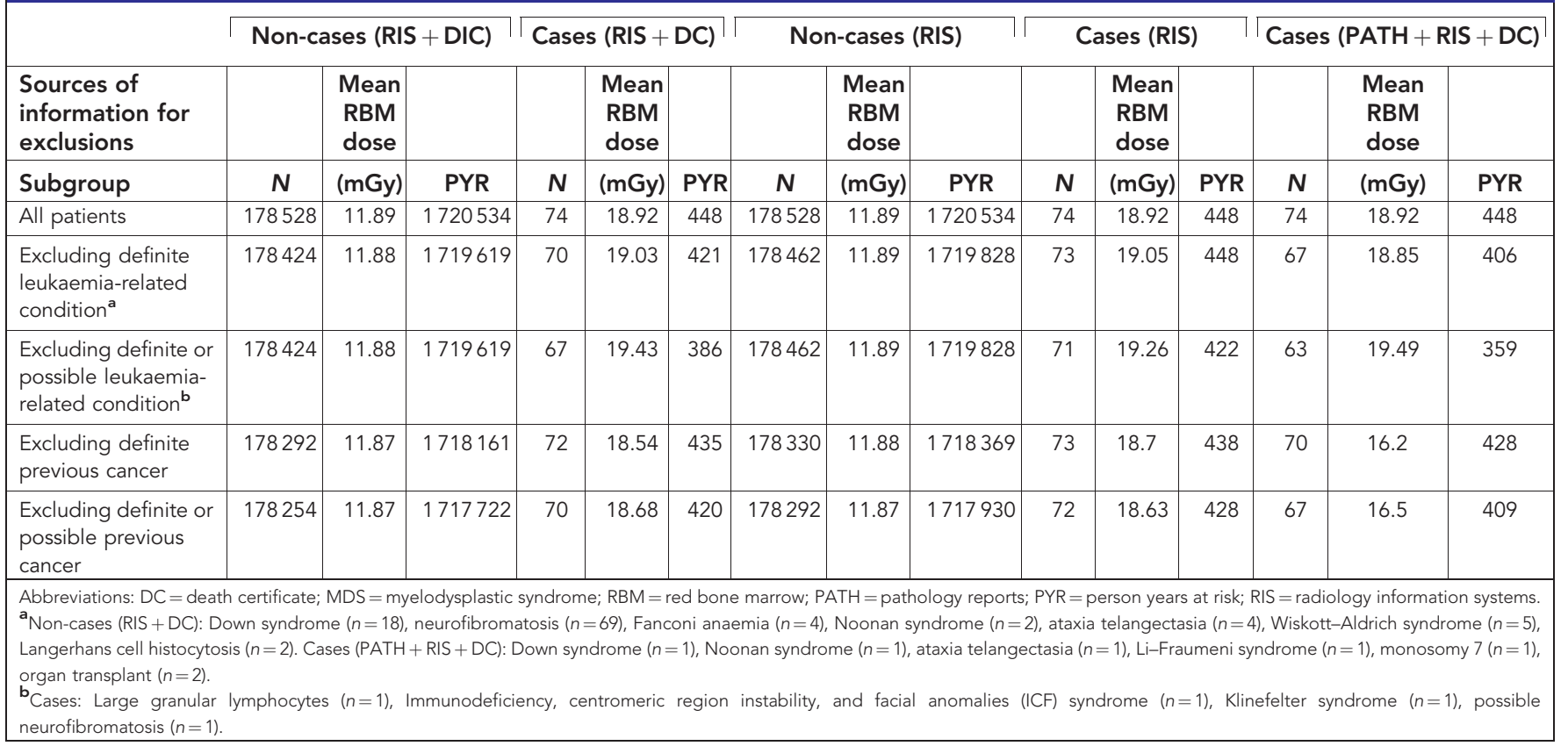

Table 3. Excess relative risk (ERR) per $\mathrm{mGy}$ for leukaemia/MDS in relation to estimated red bone marrow dose from CT scans for all cases and after exclusions

\begin{tabular}{|c|c|c|c|c|}
\hline \multirow[t]{2}{*}{ Exclusion source } & Exclusions & Cases & ERR per mGy $(+95 \% \mathrm{Cl})$ & $P$-trend \\
\hline & None & 74 & $0.036(0.005,0.118)$ & 0.01 \\
\hline \multirow[t]{4}{*}{ RIS and DC } & Leukaemia-related conditions & 70 & $0.037(0.005,0.126)$ & 0.01 \\
\hline & Leukaemia-related and possibly related conditions & 67 & $0.037(0.005,0.125)$ & 0.01 \\
\hline & Previous cancers & 72 & $0.033(0.004,0.114)$ & 0.02 \\
\hline & Previous cancers and possible previous cancers & 70 & $0.031(0.003,0.109)$ & 0.02 \\
\hline \multirow[t]{4}{*}{ RIS } & Leukaemia-related conditions & 73 & $0.041(0.007,0.135)$ & 0.01 \\
\hline & Leukaemia-related and possibly related conditions & 71 & $0.040(0.006,0.133)$ & 0.01 \\
\hline & Previous cancers & 73 & $0.035(0.004,0.121)$ & 0.01 \\
\hline & Previous cancers and possible previous cancers & 72 & $0.032(0.003,0.110)$ & 0.02 \\
\hline \multirow[t]{4}{*}{ PATH, RIS and DC } & Leukaemia-related conditions & 67 & $0.034(0.004,0.116)$ & 0.02 \\
\hline & Leukaemia-related and possibly related conditions & 63 & $0.037(0.005,0.129)$ & 0.01 \\
\hline & Previous cancers & 70 & $0.020(-0.011,0.086)^{a}$ & 0.13 \\
\hline & Previous cancers and possible previous cancers & 67 & $0.020(-0.011,0.089)^{a}$ & 0.14 \\
\hline
\end{tabular}
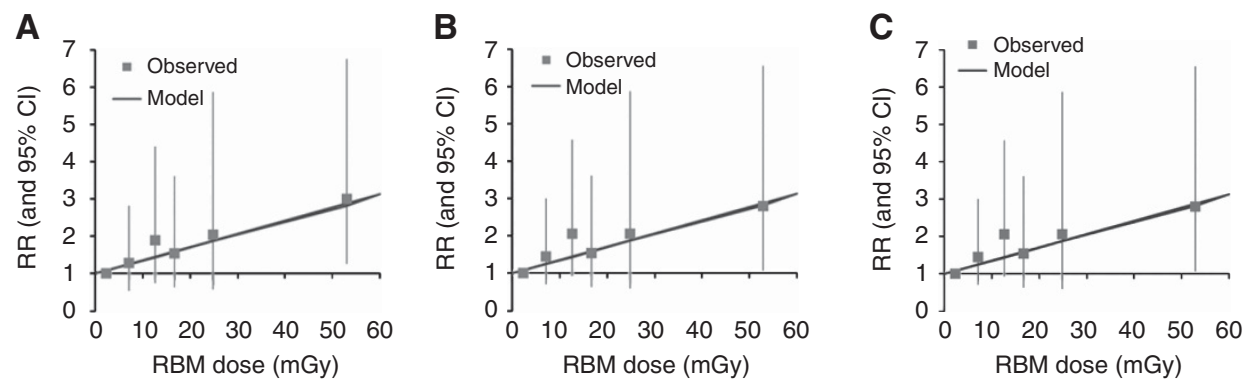

Figure 1. Dose-response relationship for leukaemia/MDS in relation to RBM dose from CT scans (exclusions based on RIS + death certificates). (A) No exclusions. (B) Excluding underlying conditions. (C) Excluding previous cancers.

biased the relationship between leukaemia or brain tumours and paediatric CT scans in our large retrospective cohort. Although a higher proportion of the cases than non-cases had cancerpredisposing conditions, these patients did not undergo more CT scans or have higher estimated radiation doses, hence these conditions did not confound the relationship. Exclusion of patients with prior cancer diagnoses that were not reported to the NHSCR did, however, reduce the ERR per $\mathrm{mGy}$ by about $15 \%$ for leukaemia and by $30 \%$ for brain tumours. The dose-response remained statistically significant, however, and the lower dose- 
Table 4. Summary of the brain tumour-related conditions, previous cancers and mean brain dose ( $m$ Gy) in brain tumour cases and non-cases

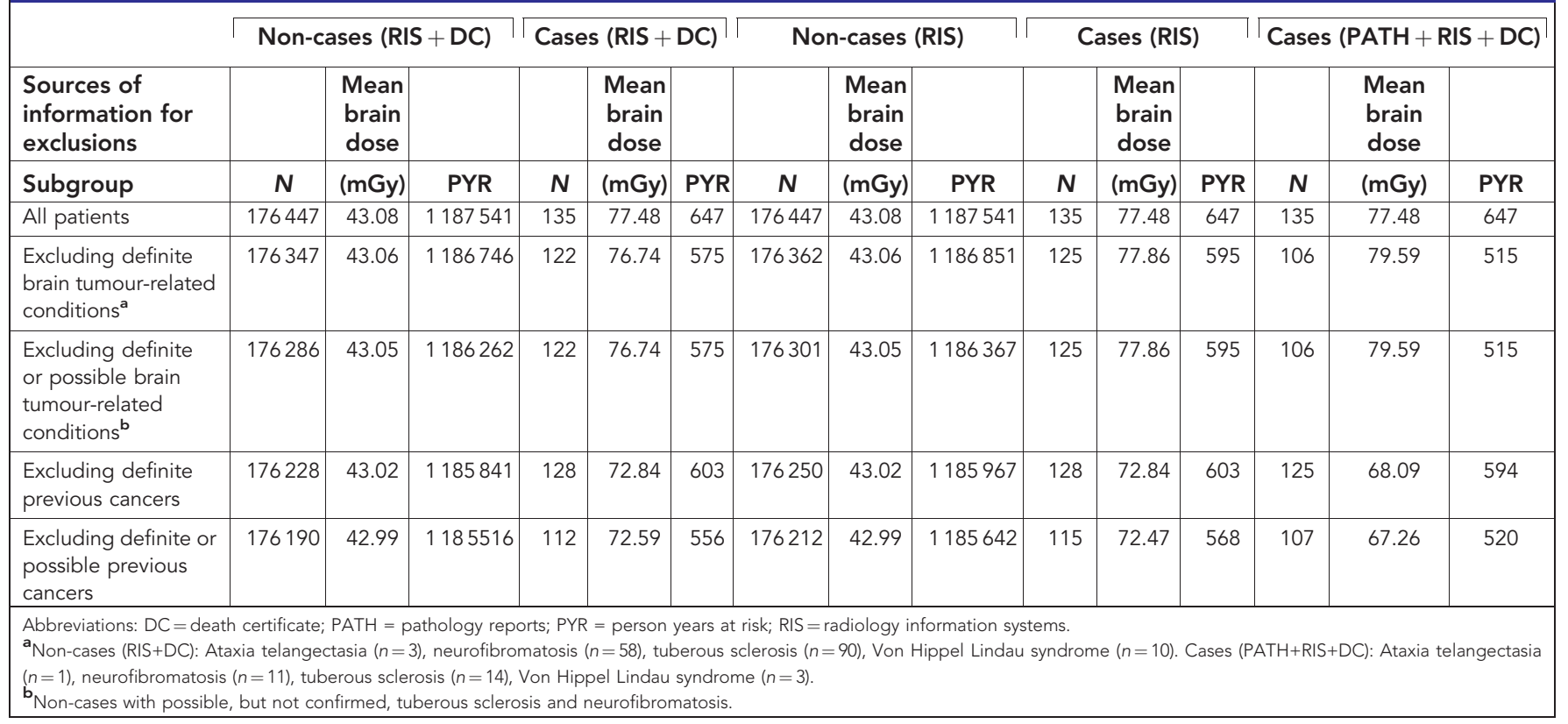

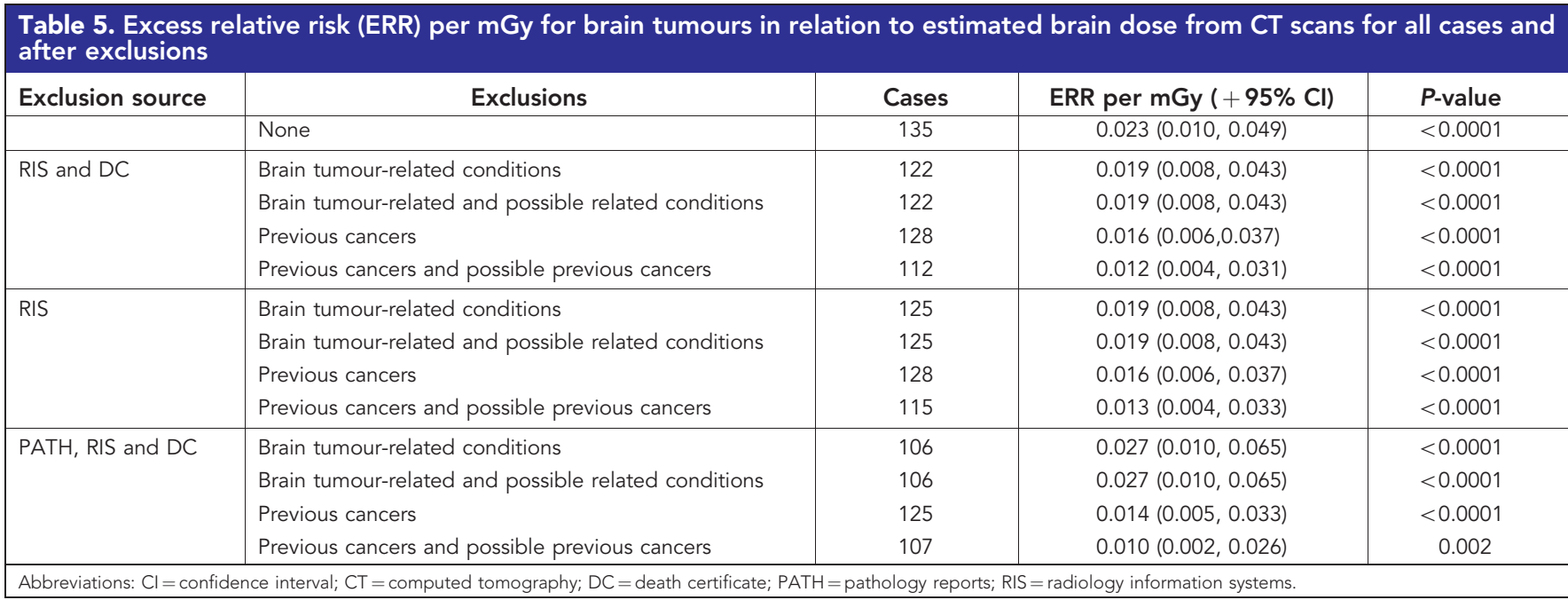

A

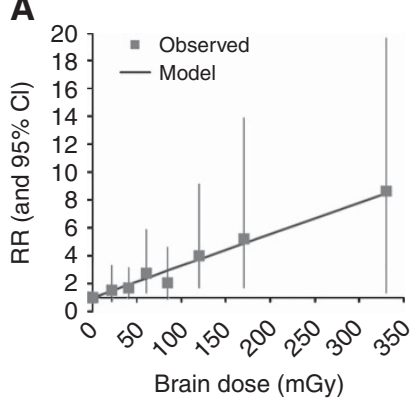

B

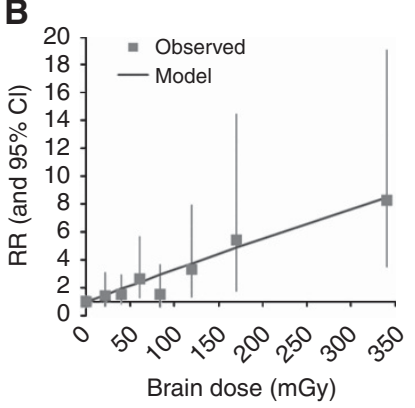

C

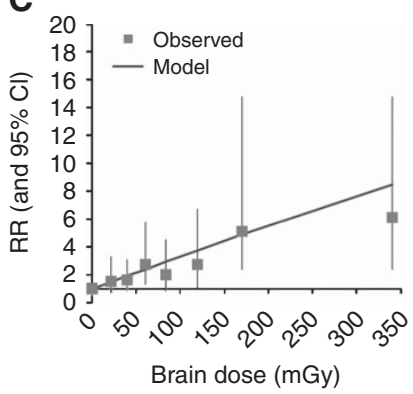

Figure 2. Dose-response relationship for brain tumours in relation to brain dose from CT scans (exclusions based on RIS + death certificates). (A) No exclusions. (B) Excluding underlying conditions. (C) Excluding previous cancers.

response for brain tumours was more compatible with the observed risk estimates for brain tumours following childhood radiation exposure in the Japanese atomic bomb survivors (Pearce et al, 2012a).
A recent evaluation of the question of underlying conditions and previous cancers was conducted in a smaller French cohort of CT scans and cancer risk ( $n=67274$; Journy et al, 2015). There was no evidence that children with underlying leukaemia-related 
conditions had higher RBM dose than those without, but estimated brain dose was higher in the children with conditions predisposing to brain tumours. The estimated ERR per mGy for leukaemia was reduced slightly after exclusion of underlying conditions (0.057 vs 0.047), but case numbers were small $(n=25)$ and the risk was not significant before or after exclusions. For brain tumours and lymphomas, the ERR per mGy was halved (0.022 vs 0.012 and 0.018 vs 0.008 , respectively) but was also non-significant $(n=27$ brain tumours, $n=21$ lymphomas). There are several major differences that complicate direct comparison with our study, particularly the different calendar periods of exposure: 1980-2002 in the United Kingdom vs 2000-2010 in France. CT scan use may have increased in children with Down syndrome more recently as scan times have reduced. The calendar period difference could explain the higher proportion of cases with Down syndrome in the French cohort and lower average RBM doses. In addition, their study population came from a number of specialist hospitals, which resulted in an enriched population for serious conditions and finally they used hospital inpatient data to ascertain the clinical conditions. They also used a somewhat different list of cancerpredisposing conditions.

In a German cohort $(n=44584)$, within the subset with additional clinical information, none of their seven cases of leukaemia and only one of the six cases of CNS tumours had underlying conditions or signs of preexisting cancer on CT (Krille et al, 2015). Exclusions, therefore, did not materially impact the results for these outcomes. A larger proportion of the lymphomas (5 out of 13) had underlying conditions, particularly posttransplant lymphoproliferative disease $(n=4)$ and 4 of the 13 cases with other solid cancers (non-CNS) had evidence of the cancer on the first CT scan. This suggests that careful review is needed of these other solid cancers in future studies to minimise potential bias. A number of other cohorts are in progress and several are collecting similar clinical information, so future comparison will be possible. The differences between the UK, French and German cohorts demonstrate the dangers of generalising the impact of these potential biases across populations and calendar periods.

We found evidence of under-ascertainment of cancers in the NHSCR. Although the degree of under-ascertainment was generally $<10 \%$, it did have an impact on our risk estimates, as these patients had more CT scans than the other patients possibly owing to the previous cancer. We could not confirm that the CT scans were taken because of the previous cancer in all instances, because our clinical information did not enable us to ascertain the date of diagnosis of the previous cancer. The pathology reports were the key source of information on these previous cancers in the cases, and we would recommend collection and review of pathology reports in other ongoing studies of CT scans and cancer risk. We did not base our primary analysis on exclusions from pathology reports, however, because they were not available for non-cases. Benign brain tumours are reportable in the United Kingdom, but it is suspected that they are under-reported, and cases who undergo surgery are more likely to be reported (Pobereskin, 2001). Our review of the radiologists' comments did reveal a number of brain tumours that were being monitored by CT and were not reported to NSHCR or were reported at a much later date. This is an issue that needs to be carefully considered in other ongoing cohorts. If benign brain tumours are not reportable in some countries, then it is unclear whether malignant brain tumours can be studied in relation to CT scan risk, because these benign brain tumours are likely to introduce confounding owing to the increased risk of a subsequent malignant brain tumour (possibly from the treatment of the benign tumour) and higher number of CT scans.

We still do not know what the causes are of most childhood leukaemias (Greaves, 2006). It is possible, therefore that there is residual confounding by unknown factors. The most widely accepted theory currently is that common childhood infections could be an important trigger factor for childhood leukaemia along with population mixing (Greaves, 2006). These infections are probably not serious enough to result in CT scans, although we did also assess this possibility in the radiologists' comments. Molecular characterisation of childhood leukaemias does not suggest that there are any remaining unidentified conditions that confer a high risk of the disease (Inaba et al, 2013). In contrast to the unreported brain tumours, we did not find evidence of unreported cases of MDS, which was reassuring.

We had several experts conduct this rigorous manual review of the available clinical information. Overall, we had additional clinical information for a high percentage of the cases (nearly $90 \%$ ), and where we had multiple sources for the same individual the majority confirmed the condition. Although cancer-predisposing conditions may have been missed even in patients with clinical information, these missing conditions should not have biased our results because there was little evidence that they were related to more CT scans, assuming (as is plausible) that data were missing at random. Because, by definition, pathology reports were not available for patients without cancer, we only had additional clinical information for about $40 \%$ of the non-cases. The sensitivity analysis that included information from pathology reports for cases could be interpreted as a lower bound for our risk estimates, if we assume that there were no unreported conditions/cancers in $60 \%$ of the non-cases who were lacking clinical information. In previous studies, up to $10 \%$ of cancers from the 1970s and 1980s were not ascertained in the NHSCR (Hawkins and Swerdlow, 1992; Dickinson et al, 2001). Although it may be reasonable to expect ascertainment to have improved since then, the proportion of unreported cancers that we located in our non-cases (1990s-2000s) was so much lower $(0.1-0.2 \%)$ as to suggest that we have still missed cancers in the non-cases. This suggests that there were likely to be unreported cancers in the non-cases and, therefore, this sensitivity analysis likely underestimated the risks.

The proportion of leukaemia/MDS cases in our cohort with underlying conditions was small, as expected based on previous analyses of childhood cancer registries (Narod et al, 1991). We expected that the number of leukaemia cases in our cohort with Down syndrome would be low, because CT scans were avoided in the past in these patients because of the need for sedation (personal communication with Down syndrome society). This finding may not apply to cohorts that include patients scanned more recently. In contrast, patients with underlying conditions related to radiosensitivity are now less likely to undergo CT scans, and so the proportion of patients with these conditions may be lower in patients scanned more recently. For example, MRI has largely replaced CT for patients with neurofibromatosis (Dombi et al, 2013). Our results cannot be generalised to other cancer outcomes, such as lymphomas where the degree of confounding may be different because of different underlying conditions and an increased probability of reverse causation as suggested in the French and German cohorts (Journy et al, 2015; Krille et al, 2015). We are conducting a separate investigation of these questions for lymphomas. We are also in the process of conducting further dosimetry work for a subset of patients and incorporating dose uncertainty into the risk estimates, so the revised risk estimates in the current paper may still be subject to change.

In conclusion, we found no convincing evidence that patients with underlying cancer-predisposing conditions, such as Down syndrome, resulted in bias in our risk estimates. Previous, unreported cancers may have, however, introduced some bias. After correction for this bias, there was still evidence of a relationship between radiation exposure from CT scans and the risk of these cancers. Although the magnitude of the risk was halved for brain tumours, this made our risk estimates more 
compatible with results from the Japanese atomic bomb survivors (Pearce et al, 2012a). Our findings suggest that other ongoing studies of this relationship need to review the reliability of the cancer registrations carefully, as this is a potential source of confounding. Collecting this clinical information for these large record linkage studies is complex, and the sources are likely to vary in different countries, but pathology reports and death certificates should be collected wherever possible.

\section{ACKNOWLEDGEMENTS}

This work was funded by the NIH Intramural Research Program, Cancer Research UK and the UK Department of Health.

\section{CONFLICT OF INTEREST}

The authors declare no conflict of interest.

\section{REFERENCES}

Bosticardo M, Marangoni F, Aiuti A, Villa A, Grazia Roncarolo M (2009) Recent advances in understanding the pathophysiology of WiskottAldrich syndrome. Blood 113: 6288-6295.

Burroughs L, Woolfrey A, Shimamura A (2009) Shwachman-Diamond syndrome: a review of the clinical presentation, molecular pathogenesis, diagnosis, and treatment. Hematol Oncol Clin North Am 23: 233-248.

Churpek JE, Larson RA (2013) The evolving challenge of therapy-related myeloid neoplasms. Best Pract Res Clin Haematol 26: 309-317.

Dickinson HO, Salotti JA, Birch PJ, Reid MM, Malcolm A, Parker L (2001) How complete and accurate are cancer registrations notified by the National Health Service Central Register for England and Wales? J Epidemiol Community Health 55: 414-422.

Dombi E, Ardern-Holmes SL, Babovic-Vuksanovic D, Barker FG, Connor S, Evans DG, Fisher MJ, Goutagny S, Harris GJ, Jaramillo D, Karajannis MA, Korf BR, Mautner V, Plotkin SR, Poussaint TY, Robertson K, Shih CS, Widemann BC (2013) Recommendations for imaging tumor response in neurofibromatosis clinical trials. Neurology 81: S33-S40.

Egeler RM, Neglia JP, Puccetti DM, Brennan CA, Nesbit ME (1993) Association of Langerhans cell histiocytosis with malignant neoplasms. Cancer 71: 865-873.

Engels EA, Pfeiffer RM, Fraumeni Jr JF, Kasiske BL, Israni AK, Snyder JJ, Wolfe RA, Goodrich NP, Bayakly AR, Clarke CA, Copeland G, Finch JL, Fleissner ML, Goodman MT, Kahn A, Koch L, Lynch CF, Madeleine MM, Pawlish K, RAO C, Williams MA, Castenson D, Curry M, Parsons R, Fant G, Lin M (2011) Spectrum of cancer risk among US solid organ transplant recipients. JAMA 306: 1891-1901.

Frush DP, Goske MJ (2015) Image gently: toward optimizing the practice of pediatric CT through resources and dialogue. Pediatr Radiol 45: 471-475.

Greaves M (2006) Infection, immune responses and the aetiology of childhood leukaemia. Nat Rev Cancer 6: 193-203.

Hawkins MM, Swerdlow AJ (1992) Completeness of cancer and death followup obtained through the National Health Service Central Register for England and Wales. Br J Cancer 66: 408-413.

Inaba $\mathrm{H}$, Greaves M, Mullighan CG (2013) Acute lymphoblastic leukaemia. Lancet 381: 1943-1955.
Journy N, Rehel JL, Ducou Le Pointe H, Lee C, Brisse H, Chateil JF, Caer-Lorho S, Laurier D, Bernier MO (2015) Are the studies on cancer risk from CT scans biased by indication? Elements of answer from a large-scale cohort study in France. Br J Cancer 112: 185-193.

Krille L, Dreger S, Schindel R, Albrecht T, Asmussen M, Barkhausen J, Berthold JD, Chavan A, Claussen C, Forsting M, Gianicolo EA, Jablonka K, Jahnen A, Langer M, Laniado M, LOTZ J, Mentzel HJ, Queisser-Wahrendorf A, Rompel O, Schlick I, Schneider K, Schumacher M, Seidenbusch M, Spix C, Spors B, Staatz G, Vogl T, Wagner J, Weisser G, Zeeb H, Blettner M (2015) Risk of cancer incidence before the age of 15 years after exposure to ionising radiation from computed tomography: results from a German cohort study. Radiat Environ Biophys 54: 1-12.

Liew E, Owen C (2011) Familial myelodysplastic syndromes: a review of the literature. Haematologica 96: 1536-1542.

Lindor NM, Mcmaster ML, Lindor CJ, Greene MH (2008) Concise handbook of familial cancer susceptibility syndromes-second edition. J Natl Cancer Inst Monogr, pp 1-93.

Machatschek JN, Schrauder A, Helm F, Schrappe M, Claviez A (2004) Acute lymphoblastic leukemia and Klinefelter syndrome in children: two cases and review of the literature. Pediatr Hematol Oncol 21: 621-626.

Metayer C, Curtis RE, Vose J, Sobocinski KA, Horowitz MM, Bhatia S, Fay JW, Freytes CO, Goldstein SC, Herzig RH, Keating A, Miller CB, Nevill TJ, Pecora AL, Rizzo JD, Williams SF, Li CY, Travis LB, Weisdorf DJ (2003) Myelodysplastic syndrome and acute myeloid leukemia after autotransplantation for lymphoma: a multicenter case-control study. Blood 101: 2015-2023.

Mettler Jr FA, Bhargavan M, Faulkner K, Gilley DB, Gray JE, Ibbott GS, Lipoti JA, Mahesh M, Mccrohan JL, Stabin MG, Thomadsen BR, Yoshizumi TT (2009) Radiologic and nuclear medicine studies in the United States and worldwide: frequency, radiation dose, and comparison with other radiation sources1950-2007. Radiology 253: 520-531.

Narod SA, Stiller C, Lenoir GM (1991) An estimate of the heritable fraction of childhood cancer. Br J Cancer 63: 993-999.

Pearce MS, Salotti JA, Little MP, Mchugh K, Lee C, Kim KP, Howe NL, Ronckers CM, Rajaraman P, Craft AW, Parker L, Berrington De Gonzalez A (2012a) Radiation exposure from CT scans in childhood and subsequent risk of leukaemia and brain tumours: a retrospective cohort study. Lancet 380: 499-505.

Pearce MS, Salotti JA, Little MP, Mchugh K, Lee C, Kim KP, Howe NL, Ronckers CM, Rajaraman P, Craft AW, Parker L, Berrington De González AB (2012b) CT scans in childhood and risk of leukaemia and brain tumoursAuthors' reply. Lancet 380: 1736-1737.

Pobereskin LH (2001) The completeness of brain tumour registration in Devon and Cornwall. Eur J Epidemiol 17: 413-416.

Seewald L, Taub JW, Maloney KW, Mccabe ER (2012) Acute leukemias in children with Down syndrome. Mol Genet Metab 107: 25-30.

Shiels MS, Pfeiffer RM, Gail MH, Hall HI, Li J, Chaturvedi AK, Bhatia K, Uldrick TS, Yarchoan R, Goedert JJ, Engels EA (2011) Cancer burden in the HIV-infected population in the United States. J Natl Cancer Inst 103: 753-762.

Ward AC, Dale DC (2009) Genetic and molecular diagnosis of severe congenital neutropenia. Curr Opin Hematol 16: 9-13.

This work is published under the standard license to publish agreement. After 12 months the work will become freely available and the license terms will switch to a Creative Commons AttributionNonCommercial-Share Alike 4.0 Unported License.

Supplementary Information accompanies this paper on British Journal of Cancer website (http://www.nature.com/bjc) 